\title{
A Hybrid Routing Approach for Opportunistic Networks
}

\author{
Ling-Jyh Chen \\ Chen-Hung Yu \\ Tony Sun \\ Yung-Chih Chen \\ Hao-hua Chu \\ Academia Sinica National Taiwan University UCLA \\ Academia Sinica \\ cclljj@iis.sinica.edu.twr94922140@ntu.edu.tw tonysun@cs.ucla.edu ycchen@iis.sinica.edu.tw \\ National Taiwan University \\ haochu@ntu.edu.tw
}

\begin{abstract}
With wireless networking technologies extending into the fabrics of our working and operating environments, proper handling of intermittent wireless connectivity and network disruptions is of significance. As the sheer number of potential opportunistic application continues to surge (i.e. wireless sensor networks, underwater sensor networks, pocket switched networks, transportation networks, and etc.), the design for an effective routing scheme that considers and accommodates the various intricate behaviors observed in an opportunistic network is of interest and remained desirable. While previous solutions use either replication or coding techniques to address the challenges in opportunistic networks, the tradeoff of these two techniques only make them ideal under certain network scenarios. In this paper, we propose a hybrid scheme, named H-EC, to deal with a wide variety of opportunistic network cases. $\mathrm{H}-\mathrm{EC}$ is designed to fully combine the robustness of erasure coding based routing techniques, while preserving the performance advantages of replication techniques. We evaluate $\mathrm{H}-\mathrm{EC}$ against other similar strategies in terms of delivery ratio and latency, and find that $\mathrm{H}$-EC offers robustness in worst-case delay performance cases while achieving good performance in small delay performance cases. We also discuss the traffic overhead issues associated with H-EC as compared to other schemes, and present several strategies that can potentially alleviate the traffic overhead of H-EC schemes.
\end{abstract}

\section{Categories and Subject Descriptors}

C.2.2 [Network Protocols]: Routing protocols

\section{General Terms}

Algorithms, Performance, Theory.

\section{Keywords}

Erasure Coding, Hybrid Approach, Opportunistic Networks, Routing.

Permission to make digital or hard copies of all or part of this work for personal or classroom use is granted without fee provided that copies are not made or distributed for profit or commercial advantage and that copies bear this notice and the full citation on the first page. To copy otherwise, to republish, to post on servers or to redistribute to lists, requires prior specific permission and/or a fee.

SIGCOMM'06 Workshops September 11-15, 2006, Pisa, Italy.

Copyright 2006 ACM 1-59593-417-0/06/0009 ...\$5.00.

\section{INTRODUCTION}

With prevalent adaptation and usage of wireless communication technologies, networking challenges evolve when continuous network connectivity cannot be guaranteed. For these challenged networking environments such as found in mobile in-motion networks and dynamic wireless networks, network connectivity are rather opportunistic in nature. Techniques for making applications tolerant to disruptions and/or high delays are essential for these opportunistic networks.

Opportunistic networks is a type of challenged networks, where communication opportunities (contacts) are intermittent, an end-to-end path between the source and the destination may have never existed, disconnection and reconnection is common, or link performance is highly variable or extreme. Therefore, traditional MANET and Internet routing techniques can not be directly applied towards networks in this category. With numerous emerging opportunistic networking applications, such as wireless sensor networks (WSN) [3, 26], underwater sensor networks (UWSN) [8], pocket switched networks (PSN) $[6,7,14]$, people networks $[10,22,24]$, transportation networks $[2,4,16]$, and etc., it remains desirable/necessary to develop an effective routing scheme that can better accommodate the various characteristics of opportunistic networks.

Several routing schemes have been previously proposed for opportunistic network scenarios [4, 12, 17, 19, 23, 25, 28], all aimed at resolving specific routing problems in opportunistic networks. These routing schemes can be grouped into two main categories according to their basic technical strategies, which are replication based and coding based.

Replication based routing schemes are the most popular design choice in existing opportunistic routing schemes. The basic idea of replication based schemes is to inject multiple identical copies of data into the network, and rely on node mobility to disseminate the data toward the destination [23]. A message is regarded to be successfully delivered when at least one of the multiple copies is received by the destination. Intuitively, if the number of replica in the network is sufficiently large, replication based schemes tend to achieve the best delay performance (i.e., the shortest delivery latency) in opportunistic networks. However, the main drawback of this type of schemes is the tremendous traffic overhead associated with flooding data replicates. As a result, when network resources (e.g., buffer space and network bandwidth) are limited, replication based schemes tend to degrade performance reliability (i.e, the delivery ratio) unless additional overhead reduction strategies are in place to alleviate traffic overhead $[4,12,17,19]$. 
For coding based routing schemes, a message (or a group of messages) are transformed into another format prior to transmission [25, 28]. The design principle of coding based schemes is to embed additional information (e.g., redundancy [25] or decoding algorithm [28]) within the coded blocks such that the original message can be successfully reconstructed with only a certain number of the coded blocks. More precisely, differing from replication based schemes which rely on successful delivery of each individual data block, coding based schemes would consider a block successfully delivered, when the necessary number of blocks are received to reconstruct the original data, this can just be a small portion of the total number of the blocks transmitted. As a result, coding based schemes tend to be more robust than replication based schemes when the network connectivity is extremely poor (this is considered as the worst delay performance cases). However, coding based schemes are less efficient when the network is well connected (this is considered as the very small delay performance cases), which is simply due to additional information embedded in the code blocks.

Nevertheless, a successful routing technique in opportunistic networks must consider both performance and reliably. An effective routing scheme that can accommodate both worst delay performance and very small delay performance cases remained challenging and desirable. In this paper, we propose a hybrid routing scheme, called H-EC, to proficiently deliver messages in various opportunistic network scenarios. H-EC is designed to fully combine the robustness of coding based routing techniques, while preserving the performance advantages of replication techniques. We evaluate the performance of $\mathrm{H}-\mathrm{EC}$ scheme via various simulations, and the results show that H-EC is capable of performing aggressively (i.e., achieving very short delivery latency) for very small delay performance cases and remains robust for worst delay performance cases. Moreover, we show that the overhead of H-EC scheme is moderate (compared with EC and other replication based schemes) and can be further reduced by applying several overhead reduction strategies.

The rest of the paper is organized as follows. In section 2, we summarize related work and recap the erasure coding based data forwarding scheme. Section 3 describes the two proposed routing schemes, namely A-EC and H-EC schemes. Section 4 presents simulation results from various opportunistic network scenarios. Section 5 discusses the overhead issue of our proposed methods and presents several strategies that can potentially reduce the routing overhead. Section 6 concludes the paper.

\section{RELATED WORK AND OVERVIEW}

\subsection{Related Work}

Routing in an opportunistic network is challenging and remains quite unique from conventional network routing methods. An ideal routing scheme in opportunistic networks has to provide reliable data delivery even when the network connectivity is intermittent or when an end-to-end path is temporally nonexistent. Moreover, since 'contacts' in an opportunistic network may appear arbitrarily without prior information, neither scheduled optimal routing (e.g., linear programming routing in DTN of scheduled contacts [15]) nor mobile relay approaches (e.g., Message Ferrying [29, 30]) can be applied.
For opportunistic networks, replication is the most popular design choice in existing opportunistic routing schemes. For instance, the Epidemic Routing scheme [23] sends identical copies of a message simultaneously over multiple paths to mitigate the effects of a single path failure, thus increases the possibilities of successful message delivery. However, flooding duplicate data tends to be very costly in terms of traffic overhead and energy consumption.

To address the excess traffic overhead from flooding replicate data, a Controlled Flooding scheme has been proposed to reduce the flooding cost while keeping the delivery reliable [12]. In this scheme, the message flooding is controlled by three parameters, namely willingness probability, Timeto-Live, and Kill Time. Additionally, once the receiver successfully receives a message, a Passive Cure is generated to "heal" the nodes in the network after they have been "infected" by that message. Therefore, with the ability to resolve excess amount of traffic overhead while provides reliable data delivery, controlled flooding scheme greatly relieves the network overhead .

Node mobility also impacts the effectiveness of opportunistic routing schemes. When the network mobility departs from the well-known random way-point mobility model (e.g., the Pursue Mobility Model [5] and the Reference Point Group Mobility Model [13]), previous studies have shown that the overhead carried by epidemic and/or flooding based routing schemes can be further reduced by taking into account the knowledge of node mobility. For instance, Probabilistic Routing scheme [18, 19] calculates the delivery predictability from a node to a particular destination node based on the observed contact history, and it forwards a message to its neighboring node if and only if that neighbor node has a higher delivery predictability value. This scheme has also been revised by Leguay et al [17] by taking the mobility pattern into account, i.e., a message is forwarded to the neighbor node if and only if the neighbor node has a mobility pattern more similar to the destination. [17] shows that the revised mobility pattern based scheme is more effective than previous ones.

Apart from the previous mentioned schemes, another class of opportunistic network routing schemes have been developed via encoding techniques, which transforms a message into another format prior to transmission. For instance, an integration of network coding and epidemic routing techniques has been proposed to reduce the required number of transmissions in the network [28]. Moreover, [25] proposes to combine erasure coding and the simple replication based routing method to improve the data delivery for the worst delay performance cases in opportunistic networks.

Although network coding based routing schemes are promising in reducing the number of transmissions (thus, improve routing efficiency) in a network, it may still fail in providing effective data delivery when the delivery latency is dominated by some extremely large inter-contact time (i.e., the time duration between two communication opportunities). In such extreme cases, forwarding schemes based on erasure coding would be more ideal, since the destination is able to reconstruct the message by just receiving a certain number of erasure coded blocks, instead of all transmitted data. It is the goal of this paper to use erasure coding to design an effective routing scheme that works for opportunistic networks in the worst of circumstances. 


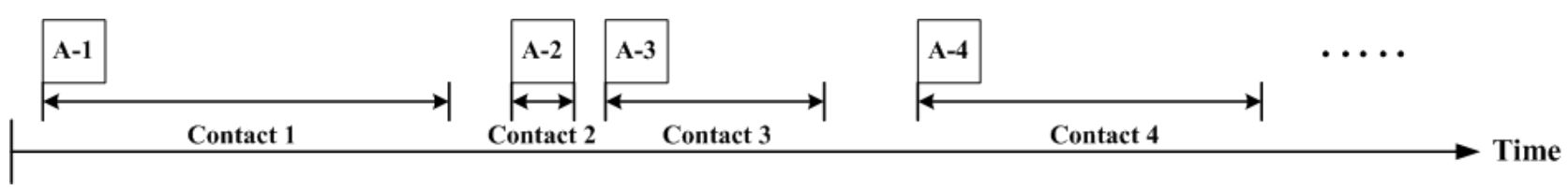

Figure 1: Illustration of the erasure coding based data forwarding algorithm (EC). In this figure, one erasure coded block $(\mathbf{A})$ is equally split among four relays $(n=4)$.

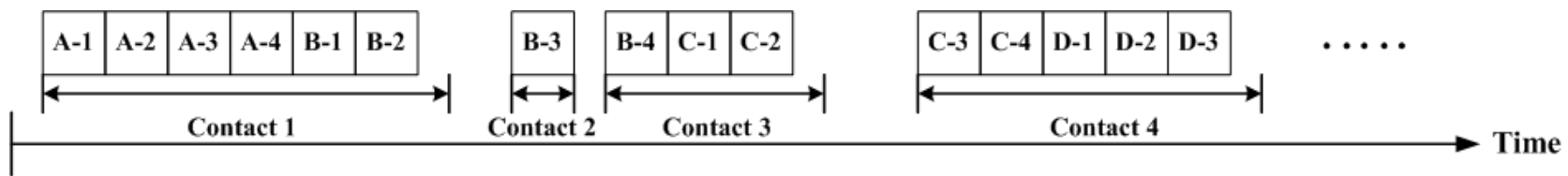

Figure 2: Illustration of the A-EC scheme, i.e., EC with aggressive forwarding. In this figure, four erasure coded blocks (A,B,C,D) are transmitted, and $n=4$.

\subsection{Overview of Erasure Code based Data For- warding}

In this subsection, we will give a brief overview on erasure code and a forwarding scheme based on erasure code, which is proposed in [25].

Erasure coding is a coding scheme which provides better fault-tolerance by adding redundancy without the overhead of strict replication, to the original data [27]. Two most popular examples of erasure coding algorithms are ReedSolomon coding and Low-Density Parity-Check (LDPC) based coding (e.g., Gallager codes, Tornado codes, and IRA codes) $[20,21]$. These algorithms differ in the encoding/decoding efficiency, replication factor, and the minimum number of code blocks to reconstruct a message. The selection of the proper erasure coding algorithm is not within the scope of this paper, and our work is based on the generic erasure coding concept.

In generic erasure coding schemes, suppose a message is of size $M$ bytes, the replication factor of erasure coding is $r$, and the coded message is fragmented into several blocks of identical size $b$ bytes, one can obtain the number of the coded blocks by $N=\frac{M \times r}{b}$. Moreover, this message can be successfully reconstructed as long as $\frac{1}{r}$ of the coded blocks are received (i.e., the minimal number of coded blocks for successfully reconstructing the message is $N / r$, which is equal to $M / b)$.

In [25], an erasure code based forwarding algorithm (EC) is proposed as illustrated in Fig. 1. In this scheme, the erasure coded blocks are equally split among $n$ relays, and relays are only allowed to send messages directly to the destination (i.e., the well-known "two-hop" scenario as used in $[6,11])$. Each relay forwards the same amount of code blocks (no duplicates in each relay), and the number of blocks forwarded by each relay can be obtained by

$$
\frac{N}{n}=\frac{M r}{b n}
$$

As reported in [25], the EC scheme is capable of providing the best worst-case delay performance with a fixed amount of overhead. However, the drawback of EC scheme is that it can not provide good very small delay performance while comparing to other popular replication based approaches.

\section{EFFECTIVE ROUTING APPROACHES FOR OPPORTUNISTIC NETWORKS}

In this section, we will discuss issues of effective routing in opportunistic networks. Ideally, an effective routing scheme not only has to provide better delivery latency in both worst delay performance and very small delay performance cases, but it also needs to remain moderate without injecting too much traffic overhead to the network. In the following subsections, we first present the routing algorithm, called A-EC, by extending EC algorithm with the aggressive forwarding technique in subsection 3.1. We then describe our proposed hybrid routing algorithm, called H-EC, in subsection 3.2.

\subsection{EC with Aggressive Forwarding}

As we have mentioned earlier, the EC scheme has attracted attentions from researchers due to its abilities to achieve better worst case delay performance in opportunistic networks; whereas, in most non-worst cases, the resulting prolonged overall delivery latency is the main drawback of the EC scheme.

The reason for such inefficiency in the EC scheme concerning non-worst delay performance cases lies in its block allocation method. In EC scheme, the number of transmitting blocks in each contact is a fixed number (i.e., $\frac{M r}{b n}$, in accordance with Eq. 1) regardless of the length of each contact duration. As a result, EC scheme can only effectively utilize each network contact when the contact duration is not much longer than the required time for sending the relayed data. If most network contact is much larger than the required time, EC scheme tends to waste the residual contact duration and thus results in ineffectiveness as illustrated in Fig. 1.

Aiming at this problem, one can easily adapt the EC scheme with an aggressive forwarding feature, which does its best to fully utilize each contact opportunity. The adapted scheme is called A-EC scheme, and we illustrate the scheme in Fig. 2.

As shown in Fig. 2, the source sends as many coded blocks as possible during each contact (totally $\frac{M r}{b n}$ blocks, i.e., $\frac{M r}{n}$ bytes). As a result, for very small delay performance cases, A-EC scheme is able to better utilize the network contact and thus expected to outperform EC scheme. 


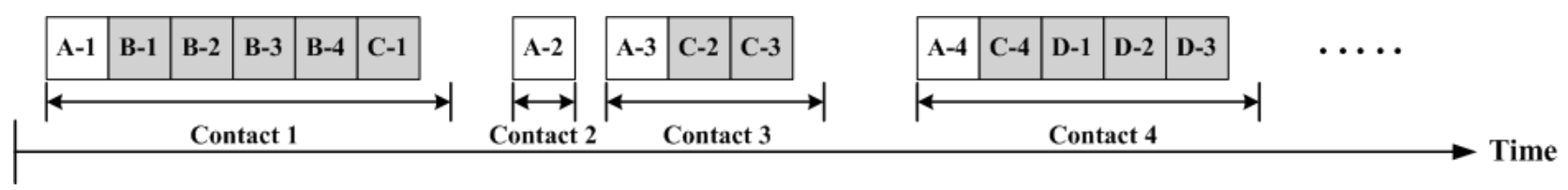

Figure 3: Illustration of H-EC scheme. In this figure, two copies of four erasure coded blocks (A,B,C,D) are transmitted: the first copy of EC blocks (the white blocks) is sent using EC algorithm, and the second copy (the gray ones) is sent using A-EC algorithm in the residual contact duration. Each coded block is equally split into 4 sub-blocks $(n=4)$.

However, for worst delay performance cases, A-EC tends to yield poor delivery ratio and/or very large delivery delay when most employed relays are either unreliable (e.g., with very limited battery power and/or buffer size) or hardly moving closer towards the destination. We call these relays black-hole nodes. As we will discuss in more details in the next section, aggressive forwarding on black-hole nodes significantly degrades the overall performance of the message delivery.

\subsection{The Hybrid Approach: H-EC}

In this subsection, we propose a hybrid erasure coding based forwarding technique, which takes advantages of both $\mathrm{EC}$ and A-EC schemes in order to achieve better message delivery performance in both worst delay performance and very small delay performance cases. The proposed hybrid scheme is abbreviated as H-EC. H-EC scheme is illustrated in Fig. 3.

More precisely, in H-EC scheme, two copies of EC blocks (constructed based on the erasure coding and replication technique described in subsection 2.2) are transmitted by the sender. The first copy of EC blocks is sent similar to how the original EC scheme does (shown as the white blocks in Fig. 3), and the second EC block is sent using A-EC algorithm during the residual contact duration after sending the first EC block (shown as the gray blocks in Fig. 3). For general opportunistic network scenarios (i.e., without blackhole nodes), H-EC scheme is expected to better utilize each contact opportunity (i.e., due to A-EC features); whereas, while black-hole nodes are present in the network, H-EC scheme is expected to perform similarly to EC scheme, which provides better forwarding performance in the worst delay performance cases.

It should also be mentioned that the traffic overhead carried by $\mathrm{H}-\mathrm{EC}$ is indeed moderate. In fact, since only two copies of EC blocks are sent by the source, the total amount of traffic consumed by $\mathrm{H}-\mathrm{EC}$ scheme is just twice of the EC scheme, which should be acceptable in most network scenarios.

\section{EVALUATION}

We perform a set of simulation to evaluate the delay performance of A-EC and H-EC schemes in two different opportunistic network scenarios. We implement EC, A-EC, and $\mathrm{H}-\mathrm{EC}$ schemes and run simulations in the java based DTN simulator, DTNSIM [1]. Moreover, we implement a simple replication based EC scheme (called R-EC), which sends EC blocks using srep algorithm, with replication factor $k$, as described in [25], such that the total traffic amount of R-EC scheme is $k$ times of the traffic amount of EC scheme.
The opportunistic network scenarios examined composes of 34 nodes (one source, one destination, and 32 intermediate relays) and the buffer size on each node is configured to be infinite. Both of the inter-contact time and contact duration of the network are given power-law distributed values with coefficient 0.6 (as reported in $[6,14]$ ). Similar to the scenarios used in [25], messages are generated at a Constant Bit Rate (CBR) of 12 messages/day for 160 days (i.e., $M=12 \times 160=1920$ ), and each message is 1200 bytes in size. For all EC based schemes, the code block size, $b$, is set to 150 bytes, and the other two parameters, $r$ and $n$, are set to 2 and 16 respectively. We present evaluation results as follows.

\subsection{A-EC in general network scenarios}

First, we evaluate EC, A-EC, and R-EC ( $k=2$ and 4$)$ schemes in the general network scenario. Note that, in fact, EC scheme is an instance of R-EC scheme, with its replication factor $k$ set equal to 1 . Fig. 4 depicts the simulation results of data latency distribution in Complementary CDF (CCDF) curves.

From Fig. 4, the results clearly show that, as the replication factor $k$ increases, the delivery delay performance of $\mathrm{R}-\mathrm{EC}$ and RC schemes improves significantly. More specifically, in our simulation results, EC scheme is able to successfully deliver $50 \%$ of data within 70,000 seconds; whereas R-EC scheme could deliver $50 \%$ data within 46,000 seconds

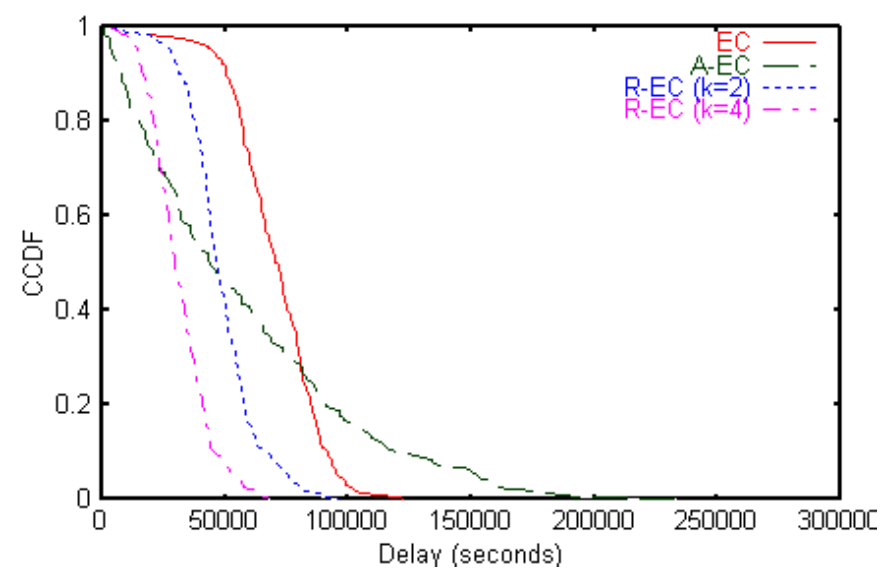

Figure 4: Latency distribution of EC, A-EC, and R-EC ( $k=2$ and 4) schemes in general network scenarios. The distribution is shown in Complementary CDF (CCDF) curve. 


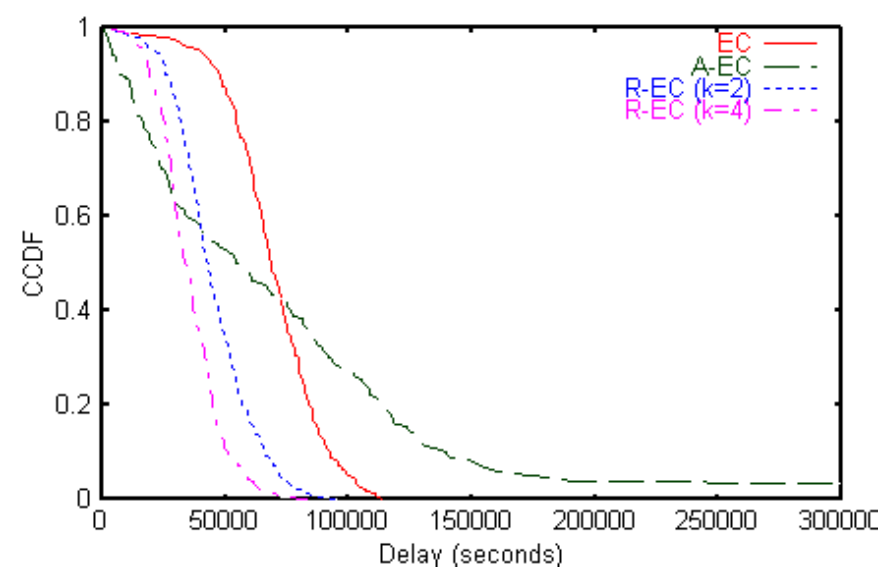

Figure 5: Latency distribution (CCDF) of EC, AEC, and R-EC ( $k=2$ and 4$)$ schemes in black-hole network scenario.

when $k=2$, and within 30,000 seconds when $k=4$. It turns out that the larger $k$ is, the better delivery performance $\mathrm{R}$ EC achieves.

On the other hand, it should also be mentioned that with a larger $k$ value, more traffic overhead will be experienced with the R-EC scheme. Therefore, it is necessary to give $k$ an upper bound, this bound turns out to be the maximum number of blocks that can be sent over during a contact duration. The performance of $\mathrm{R}-\mathrm{EC}$, as $k$ increases, will eventually converged towards the performance of A-EC scheme for the very small delay performance cases, since R-EC becomes A-EC when it completely utilizes all residual contact opportunities. In Fig. 4, while only looking at the left portion of the figure (i.e., the very small delay cases), A-EC does achieve shorter delivery latency than EC and the REC schemes. Nonetheless, as $k$ increases, the performance of R-EC scheme becomes closer to the A-EC curve, which confirms our intuition.

However, while looking at the right portion of Fig. 4, one can also observe that A-EC scheme has a longer tail than R-EC and EC schemes. The reason is that though the aggressive forwarding feature yields better utilization for each contact (and thus reduce the delivery delay), it is also very likely to fail if the selected relays have very large latency to their next contact with the destination. As a result, A-EC performs much worse than the other schemes in worst delay performance cases.

\subsection{A-EC in black-hole scenarios}

In the second experiment, we evaluate A-EC scheme in the black-hole scenario, where some of the relay nodes are extremely uncooperative (i.e., limited battery power, limited buffer size, or extremely large inter-contact time with the destination). For simplicity, we use the same simulation parameters as the one employed in the previous subsection, except that five (out of 32) relays are set as uncooperative nodes with very small buffer size (the buffer size is two messages size). Fig. 5 depicts the simulation results.

The results are basically consistent with what is shown in the previous subsection (Fig. 4), except that A-EC scheme suffers from more serious overall delivery delay in the black-

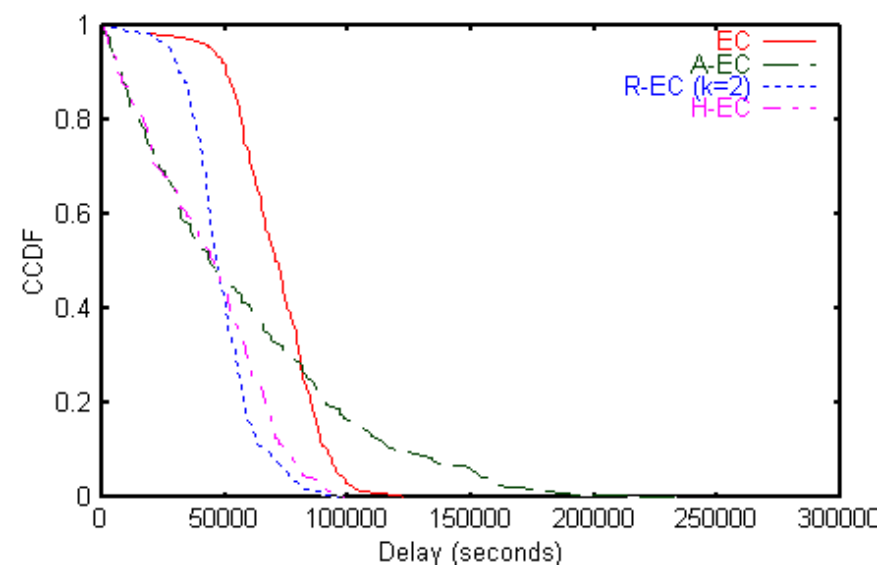

Figure 6: Latency distribution (CCDF) of EC, AEC, R-EC $(k=2)$ and H-EC schemes in general network scenarios.

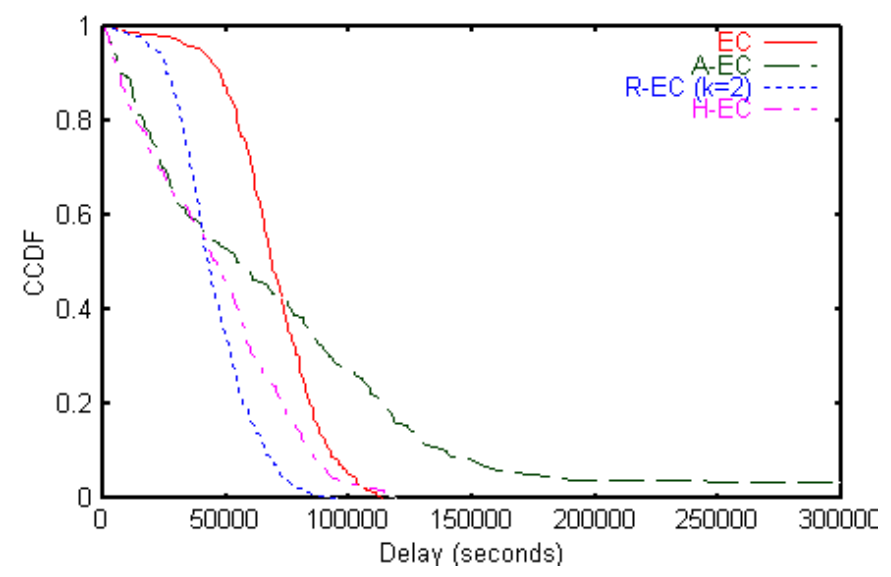

Figure 7: Latency distribution (CCDF) of EC, AEC, R-EC $(k=2)$ and H-EC schemes in black-hole network scenarios.

hole scenario, as illustrated by the heavy tail of A-EC in Fig. 5. More precisely, in the black-hole scenario, the results shows that around $5 \%$ of messages will not be successfully delivered after 300,000 seconds; whereas, in the general scenario, all messages are successfully delivered within 200,000 seconds. It turns out that, while black-hole nodes present, A-EC scheme is very likely to fail in providing reliable data delivery in opportunistic networks.

\subsection{Evaluation of $\mathrm{H}$-EC scheme}

Here, we evaluate our proposed H-EC scheme in both general and black-hole scenarios. The simulation parameters (for both cases) employed here are the same as the ones employed in previous subsections. We compare the simulation results of EC, A-EC, R-EC (with $k=2$, which has the same amount of overhead as H-EC scheme), and H-EC schemes in Fig. 6 and 7.

In Fig. 6, the result clearly shows that, the proposed H-EC scheme always performs comparably to the optimal scheme in all test cases. More precisely, in very small de- 
lay performance cases, the performance of H-EC scheme is almost identical to the A-EC scheme; whereas in the worst delay performance cases, H-EC still performs closely (but slightly worse) to the R-EC scheme. The results indicate that, in very small delay performance cases, H-EC is able to take better advantages of network communication opportunities via a more aggressive forwarding mechanism. Moreover, in the worst delay performance cases, H-EC preserves the reliability of EC and R-EC scheme, and is able to yield better delivery performance compare to the A-EC scheme.

Additionally, for black-hole scenario, Fig. 7 shows that HEC still performs comparably to A-EC in very small delay performance cases. While in worst delay performance cases, H-EC performs closely (yet slightly better) to EC scheme (instead of R-EC scheme). It turns out that, while operating with uncooperative relay nodes, the aggressive forwarding feature of H-EC scheme does not provide advantages in data forwarding, and therefore the performance converges to the EC scheme and the overall delay increases.

\section{DISCUSSION}

In this section, we examine the overhead of our proposed approach in terms of the total amount of traffic injected to the network and the average energy consumption of each relay. Moreover, we propose several strategies that can potentially reduce the overhead carried by our proposed routing scheme. Note that, this section only discusses routing schemes which are erasure coding and/or simple replication based. We will defer the evaluation on routing schemes that are based on other advance techniques (e.g., network coding based routing [28]).

\subsection{Overhead Analysis}

Here we compare routing overhead of several erasure coding and simple replication based routing approaches. For simplicity, the comparison results are normalized according to Direct Contact (DC) scheme [25]. Only optimal cases are considered for A-EC and H-EC schemes (i.e., the first contact duration is long enough to forward all coded blocks). We denote $r$ is the replication factor of the erasure coding algorithm employed, $n$ is the number of relays among which erasure code blocks are split, and $k$ is the replication factor employed in the simple replication algorithm. Table 1 shows the comparison results.

Table 1: Comparison of normalized routing overhead for several erasure coding and simple replication based routing schemes.

\begin{tabular}{|c|c|c|c|c|c|c|}
\hline Algorithms & DC & srep & EC & A-EC & R-EC & H-EC \\
\hline Overall Traffic & 1 & $k$ & $r$ & $r$ & $k r$ & $2 r$ \\
\hline Traffic/Relay/message & 1 & 1 & $\frac{r}{n}$ & $r$ & $\frac{r}{n}$ & $r, \frac{r}{n}$ \\
\hline
\end{tabular}

In Table 1, the overall traffic overhead of the simple replication based routing algorithm, srep, is actually proportional to the replication factor $k$ (which denotes the number of identical copies injected to the network). Whereas for A-EC and EC schemes, which are erasure coding based schemes distinguished by the aggressive forwarding option, the overall overhead is proportional to the replication factor $r$ of the employed erasure coding algorithm.
In addition, it is shown that the overall overhead of R-EC scheme is proportional to both $k$ and $r$, since this scheme in fact a combination of both erasure coding and simple replication techniques. For our proposed hybrid scheme, H-EC, the traffic overhead is just twice of the EC scheme, which is $2 r$, since it actually a combination of EC and A-EC schemes with a replication factor of 2 . Note that, while $k$ must have an integer value, $r$ can be an integer or a real number depending on the employed coding algorithm ${ }^{1}$. Moreover, since data transmission consumes the most energy in wireless communication, the results shown in Table 1 also can be interpreted as the amount of the overall energy consumed by the listed data forwarding algorithms.

Furthermore, Table 1 also compares the amount of forwarded traffic per relay per message, which can be interpreted as the survivability of the routing algorithm in extremely challenged environments (e.g., opportunistic networks with black-holes). Larger value indicates lower survivability of the corresponding algorithm. For instance, the forwarded traffic per relay per message of A-EC scheme is $n$ folds larger than the EC and the R-EC scheme, which indicates that A-EC scheme tends to rely on a small set of relays to forward messages to the destination. As a result, if some of the selected relays are uncooperative (e.g., with limited battery power and/or limited buffer size) or compromised (i.e., they reject to help forward data or forward wrong data), A-EC tends to work ineffectively with very large delivery latency and/or poor delivery ratio.

However, since EC and R-EC schemes distribute data among a larger number of relays, they are expected to perform be more robust to the presence of 'black-holes' (i.e., uncooperative and/or compromised relays) in the network. The proposed hybrid scheme, H-EC, is also expected to remain robust in such extremely challenged environments, since the survivability of R-EC based algorithm is preserved in the proposed hybrid scheme.

\subsection{Overhead Reduction Strategies}

As we have discussed earlier, although the proposed scheme, $\mathrm{H}-\mathrm{EC}$, is promising in providing effective data forwarding in opportunistic networks, the main drawback of the H-EC is the carried traffic overhead, which is $2 r$ folds of the original message size (as shown in Table 1). In this subsection, we present two strategies which can potentially reduce traffic overhead carried by the H-EC scheme.

\subsubsection{Explicit ACK or Passive Cure}

Since the H-EC scheme basically injects two identical copies of erasure coded blocks (with replication factor $r$ ) into the network, the traffic overhead is double of the EC scheme and $2 r$ folds of the original message size. Intuitively, the traffic overhead can be possibly reduced by 'healing' the network with receiver feedbacks upon successful receipt of a complete message. For instance, [4] and [12] have proposed Explicit $A C K s$ and Passive Cure schemes to alleviate traffic overhead carried by replication.

However, it should also be mentioned that, different from the scenarios employed in [4, 12], messages transmitted in $\mathrm{H}-\mathrm{EC}$ scheme are encoded. As a result, the implementation of either Explicit ACK or Passive Cure scheme has to take erasure coding into account, i.e., acknowledging/curing the

${ }^{1}$ For instance, $r$ is equal to $\frac{5}{4}$ if the employed erasure coding algorithm is equivalent to RAID-4 or RAID-5 algorithms. 
network $N$ erasure coded blocks at a time once $\frac{N}{r}$ of the blocks have been successfully delivered, where $N=\frac{M \times r}{b}$. In addition, the forwarding of receiver feedbacks has to be carefully controlled as well. For instance, the Time-To-Live [12], Kill Time [12], or Reverse Path Forwarding [9] like methods can be employed to reduce the traffic overhead of receiver feedbacks.

\subsubsection{Adaptive Coding and Forwarding}

In addition to the "healing" based strategies, one can also reduce traffic overhead and improve data forwarding efficiency via adaptive coding and forwarding. More precisely, by adaptive coding, one can adapt the replication factor, $r$, of the employed erasure coding scheme in accordance to the observed network status. When the network connectivity is very poor, a larger $r$ can provide better reliability for message delivery with the price of higher traffic overhead; whereas when the network connectivity is decent, choosing a smaller $r$ can greatly reduce the network overhead.

Moreover, with adaptive forwarding, one can also adapt the traffic volume. Instead of $b$ bytes in EC scheme or the maximum deliverable data size in A-EC scheme, the deliverable data size can be adjusted based on a probabilistic estimate to improve the packet delivery chances. For instance, this probabilistic estimate can be a function of the node mobility pattern [17], remaining battery life, available buffer space, and etc.

One should also note that the online measurement (and/or monitoring) of the network is required for cooperating the adaptive coding and forwarding scheme, e.g., measuring the mobility pattern, and probing the battery life and buffer space. It turns out that additional computation and traffic overhead are still necessary, thus these schemes must be carefully used and designed in order to improve the data forwarding efficiency.

\section{CONCLUSION}

An effective data forwarding scheme is essential for opportunistic networks, as communication opportunities in such challenged networks are opportunistic and precious. In this paper, we proposed a hybrid scheme, called H-EC, which combines the strength of erasure coding and the advantages of aggressive forwarding. With simulations, we evaluated the performance of H-EC, EC, and R-EC schemes in terms of delivery ratio and latency. The results indicated that $\mathrm{H}-$ EC scheme is able to perform aggressively (similar to A-EC scheme) for very small delay performance cases and remain robust (similar to EC and R-EC schemes) for worst-case delay performance cases. The performance advantages are quite remarkable when the network encompasses black-holes nodes. We also discussed several strategies that can potentially alleviate the traffic overhead carried by H-EC scheme (and other EC based ones). The effectiveness and robustness of H-EC render it an ideal solution that can go a long way toward effective data forwarding in opportunistic networks.

\section{REFERENCES}

[1] Delay tolerant network simulator. www.cs.washington.edu/homes/sushjain/pubs2/dtnsim.tar.gz.

[2] Umassdieselnet. http://prisms.cs.umass.edu/diesel/.

[3] The zebranet wildlife tracker. http://www.princeton.edu/ mrm/zebranet.html.
[4] J. Burgess, B. Gallagher, D. Jensen, and B. N. Levine. Maxprop: Routing for vehicle-based disruption-tolerant networking. In IEEE Infocom, 2006.

[5] T. Camp, J. Boleng, and V. Davies. A survey of mobility models for ad hoc network research. Wireless Communication and Mobile Computing Journal, 2(5):483-502, 2002.

[6] A. Chaintreau, P. Hui, J. Crowcroft, C. Diot, R. Gass, and J. Scott. Pocket switched networks: Real-world mobility and its consequences for opportunistic forwarding. Technical Report UCAM-CL-TR-617, University of Cambridge, Computer Laboratory, February 2005.

[7] A. Chaintreau, P. Hui, J. Crowcroft, C. Diot, R. Gass, and J. Scott. Impact of human mobility on the design of opportunistic forwarding algorithms. In IEEE Infocom, 2006.

[8] J.-H. Cui, J. Kong, M. Gerla, and S. Zhou. Challenges: Building scalable mobile underwater wireless sensor networks for aquatic applications. IEEE Network, Special Issue on Wireless Sensor Networking, May 2006.

[9] Y. Dalal and R. Metcalfe. Reverse path forwarding of broadcast packets. Communications of the ACM, 21:1040-1048, December 1978.

[10] J. A. Davis, A. H. Fagg, and B. N. Levine. Wearable computers as packet transport mechanisms in highly-partitioned ad-hoc networks. In IEEE Intl. Symp. on Wearable Computers, 2001.

[11] M. Grossglauser and D. Tse. Mobility increases the capacity of ad-hoc wireless networks. In IEEE Infocom, 2001.

[12] K. A. Harras, K. C. Almeroth, and E. M. Belding-Royer. Delay tolerant mobile networks (dtmns): Controlled flooding in sparse mobile networks. In IFIP Networking, 2005.

[13] X. Hong, M. Gerla, R. Bagrodia, and G. Pei. A group mobility model for ad hoc wireless networks. In $A C M$ International Workshop on Modeling, Analysis and Simulation of Wireless and Mobile Systems (MSWiM), 1999.

[14] P. Hui, A. Chaintreau, J. Scott, R. Gass, J. Crowcroft, and C. Diot. Pocket switched networks and human mobility in conference environments. In $A C M$ SIGCOMM Workshop on Delay Tolerant Networks, 2005.

[15] S. Jain, K. Fall, and R. Patra. Routing in a delay tolerant network. In ACM SIGCOMM, 2004.

[16] J. LeBrun, C.-N. Chuah, and D. Ghosal. Knowledge based opportunistic forwarding in vehicular wireless ad hoc networks. In IEEE VTC Spring, 2005.

[17] J. Leguay, T. Friedman, and V. Conan. Dtn routing in a mobility pattern space. In ACM SIGCOMM Workshop on Delay Tolerant Networks, 2005.

[18] A. Lindgren and A. Doria. Probabilistic routing protocol for intermittently connected networks. Technical report, draft-lindgren-dtnrg-prophet-01.txt, IETF Internet draft, July 2005.

[19] A. Lindgren, A. Doria, and O. Schelen. Probabilistic routing in intermittently connected networks. $A C M$ SIGMOBILE Mobile Computing and Communications 
Review, 7(3):19-20, July 2003.

[20] M. G. Luby, M. Mitzenmacher, M. A. Shokrollahi, and D. A. Spielman. Efficient erasure correcting codes. IEEE Transactions on Information Theory, 47(2):569-584, February 2001.

[21] J. S. Plank and M. G. Thomason. A practical analysis of low-density parity-check erasure codes for wide-area storage applications. In The International Conference on Dependable Systems and Networks. IEEE, June 2004.

[22] D. Snowdon, N. Glance, and J.-L. Meunier. Pollen: using people as a communication medium. Elsevier Computer Networks, 35(4):429-442, February 2001.

[23] A. Vahdat and D. Becker. Epidemic routing for partially-connected ad hoc networks. Technical Report CS-2000-06, Duke University, July 2000.

[24] R. Y. Wang, S. Sobti, N. Garg, E. Ziskind, J. Lai, and A. Krishnamurthy. Turning the postal system into a generic digital communication mechanism. In $A C M$ SIGCOMM, 2004.
[25] Y. Wang, S. Jain, M. Martonosi, and K. Fall. Erasure coding based routing for opportunistic networks. In ACM SIGCOMM Workshop on Delay Tolerant Networks, 2005.

[26] Y. Wang and H. Wu. Dft-msn: The delay fault tolerant mobile sensor network for pervasive information gathering. In IEEE Infocom, 2006.

[27] H. Weatherspoon and J. D. Kubiatowicz. Erasure coding vs. replication: A quantitative comparison. In The 1st International Workshop on Peer-to-Peer Systems, March 2002.

[28] J. Widmer and J.-Y. L. Boudec. Network coding for efficient communication in extreme networks. In $A C M$ SIGCOMM Workshop on Delay Tolerant Networks, 2005.

[29] W. Zhao, M. Ammar, and E. Zegura. A message ferrying approach for data delivery in sparse mobile ad hoc networks. In ACM MobiHoc, 2004.

[30] W. Zhao, M. Ammar, and E. Zegura. Controlling the mobility of multiple data transport ferries in a delay-tolerant network. In IEEE Infocom, 2005. 